\title{
About Trigonometric-polynomial Bounds of Sinc Function
}

\author{
Ramkrishna M. Dhaigude, Christophe Chesneau and Yogesh J. Bagul*
}

\begin{tabular}{l} 
Abstract \\
In this article, we establish sharp trigonometric-polynomial bounds for unnormalized sinc function. \\
\hline Keywords: Trigonometric-polynomial bounds; Cusa-Huygen's inequality; sinc function. \\
AMS Subject Classification (2020): Primary: 26D05 ; Secondary: 26D07; 26D15; 26D20. \\
*Corresponding author
\end{tabular}

\section{Introduction}

The unnormalized sinc function is defined as

$$
\operatorname{sinc} x=\frac{\sin x}{x} ; x \neq 0
$$

It finds applications in many branches of applied science, such as difference equations, approximation theory etc. We may refer the reader to [1], and the references therein. Many important quantities involving the unnormalized sinc function have no closed form and require a numerical evaluation. Hence, tractable and sharp bounds of them can be useful to determine their possible values and can help to understand the features of the related phenomena. For these reasons, the inequalities involving unnormalized sinc function have attracted attention of many searchers. One of the most remarkable inequality involving the unnormalized sinc function is given by

$$
\frac{\sin x}{x}<\frac{2+\cos x}{3} ; x \in(0, \pi / 2) .
$$

This inequality is known as Cusa-Huygen's inequality $[2-6,8,9]$ in the literature. The inequality (1.1) has been sharpened and extended in many ways (see e.g. [2-10] and the references therein) by searchers. In particular, the following sharp inequalities were established in [5].

Statement 1. [5, Theorem 2] For every $x \in(0, \pi / 2)$ we have

$$
\frac{2+\cos x}{3}-\frac{x^{4}}{180}<\frac{\sin x}{x}<\frac{2+\cos x}{3}-\frac{x^{4}}{180}+\frac{x^{6}}{3780} .
$$

The inequalities (1.2) were extended and sharpened in [4] as follows.

Statement 2. [4, Theorem 2] For every $x \in(0, \pi / 2)$ and $n \in \mathbb{N}$, the set of all natural numbers, we have

Received : 02-07-2019, Accepted : 04-02-2020 


$$
\begin{aligned}
& \frac{2+\cos x}{3}+\sum_{k=2}^{2 n}(-1)^{k+1} B(k) x^{2 k}<\frac{\sin x}{x} \\
& <\frac{2+\cos x}{3}+\sum_{k=2}^{2 n+1}(-1)^{k+1} B(k) x^{2 k}
\end{aligned}
$$

where $B(k)=2(k-1) /[3(2 k+1) !]$.

Recently in [3] the inequalities (1.3) have been sharpened further. These new sharpened inequalities are cited in the statement below.

Statement 3. [3, Theorem 8] If $m=2 n-1$ where $n \in \mathbb{N}$ and $x \in(0, \pi)$, then we have

$$
F(x)<\frac{\sin x}{x}<G(x)
$$

where

$$
F(x)=\frac{2 m+\cos x}{2 m+1}+\frac{2}{2 m+1} \sum_{k=1}^{m+1} \frac{k-m}{(2 k+1) !}(-1)^{k+1} x^{2 k}
$$

and

$$
G(x)=\frac{(2 m+2)+\cos x}{2 m+3}+\frac{2}{2 m+3} \sum_{k=1}^{m+2} \frac{k-m-1}{(2 k+1) !}(-1)^{k+1} x^{2 k} .
$$

In this paper, we aim to refine all the inequalities mentioned above. To achieve our main results, we need following power series expansions.

$$
\sin x=\sum_{k=0}^{\infty} \frac{(-1)^{k}}{(2 k+1) !} x^{2 k+1} ; x \in \mathbb{R}
$$

and

$$
\cos x=\sum_{k=0}^{\infty} \frac{(-1)^{k}}{(2 k) !} x^{2 k} ; x \in \mathbb{R}
$$

\section{Main Results}

First, we refine the lower bound of (1.2) in our first result.

Theorem 2.1. If $x \in(0, \pi)$ then we have

$$
\frac{4+\cos x}{5}-\frac{x^{2}}{15}<\frac{\sin x}{x}
$$

Proof. Let us set

$$
f(x)=5 \sin x+\frac{x^{3}}{3}-4 x-x \cos x .
$$

Then we have

$$
f^{\prime}(x)=x^{2}+x \sin x+4 \cos x-4
$$

and

$$
f^{\prime \prime}(x)=2 x-3 \sin x+x \cos x .
$$

By the inequality (1.1), we have $f^{\prime \prime}(x)>0$, implying that $f^{\prime}(x)>f^{\prime}(0)=0$, so $f(x)>f(0)=0$, which gives the desired inequality. 
The proof of Theorem 2.1 can also be given by showing $f(x)$ positive by using algorithm presented in [11, 12].

Our first result in Theorem 2.1 motivated us to further refine inequalities (1.3) and (1.4). We give this refinement in the next theorem.

Theorem 2.2. If $m=2 n-1$ where $n \in \mathbb{N}$ and $x \in(0, \pi)$, then we have

$$
\Phi(x)<\frac{\sin x}{x}<\Psi(x)
$$

where

$$
\Phi(x)=\frac{(2 m+2)+\cos x}{2 m+3}+\frac{2}{2 m+3} \sum_{k=1}^{m+1} \frac{k-m-1}{(2 k+1) !}(-1)^{k+1} x^{2 k}
$$

and

$$
\Psi(x)=\frac{(2 m+4)+\cos x}{2 m+5}+\frac{2}{2 m+5} \sum_{k=1}^{m+2} \frac{k-m-2}{(2 k+1) !}(-1)^{k+1} x^{2 k} \text {. }
$$

Proof. Let us set

$$
\begin{aligned}
f_{1}(x) & =\frac{\sin x}{x}-\Phi(x) \\
& =\frac{\sin x}{x}-\frac{2 m+2}{2 m+3}-\frac{1}{2 m+3} \cos x-\frac{2}{2 m+3} \sum_{k=1}^{m+1} \frac{k-m-1}{(2 k+1) !}(-1)^{k+1} x^{2 k} .
\end{aligned}
$$

Utilizing (1.5) and (1.6), after some calculations, we get

$$
\begin{aligned}
f_{1}(x) & =\sum_{k=0}^{\infty} \frac{(-1)^{k}}{(2 k+1) !} x^{2 k}-\frac{2 m+2}{2 m+3}-\frac{1}{2 m+3} \sum_{k=0}^{\infty} \frac{(-1)^{k}}{(2 k) !} x^{2 k} \\
& -\frac{2}{2 m+3} \sum_{k=1}^{m+1} \frac{k-m-1}{(2 k+1) !}(-1)^{k+1} x^{2 k} \\
& =-\frac{2 m+2}{2 m+3}+\frac{2}{2 m+3} \sum_{k=0}^{\infty} \frac{k-m-1}{(2 k+1) !}(-1)^{k+1} x^{2 k} \\
& -\frac{2}{2 m+3} \sum_{k=1}^{m+1} \frac{k-m-1}{(2 k+1) !}(-1)^{k+1} x^{2 k} \\
& =\frac{2}{2 m+3} \sum_{k=m+2}^{\infty} \frac{k-m-1}{(2 k+1) !}(-1)^{k+1} x^{2 k} \\
& =\frac{2}{2 m+3} x^{2 m+2} T,
\end{aligned}
$$

where

$$
T=\sum_{k=1}^{\infty} \frac{k}{(2 k+2 m+3) !}(-1)^{k+1} x^{2 k}
$$

Then, $T$ is an alternating series which is clearly convergent by Leibnitz rule and hence $T>0$. Thus, we have $f_{1}(x)>0$ and

$$
\Phi(x)<\frac{\sin x}{x} .
$$

Similarly, by setting

$$
f_{2}(x)=\Psi(x)-\frac{\sin x}{x},
$$

with the same arguments as in case of $f_{1}(x)$, we conclude that $f_{2}(x)>0$, i.e.,

$$
\frac{\sin x}{x}<\Psi(x)
$$

The proof follows from (2.3) and (2.4). 
Some particular cases of Theorem 2.2 for $x \in(0, \pi / 2)$ are given below.

By putting $n=1$ (so $m=1$ ) in (2.2), we get

$$
\frac{4+\cos x}{5}-\frac{x^{2}}{15}<\frac{\sin x}{x}<\frac{6+\cos x}{7}-\frac{2 x^{2}}{21}+\frac{x^{4}}{420}
$$

By putting $n=2$ (so $m=3$ ) in (2.2), we get

$$
\begin{aligned}
& \frac{8+\cos x}{9}-\frac{x^{2}}{9}+\frac{x^{4}}{270}-\frac{x^{6}}{22680}<\frac{\sin x}{x} \\
& <\frac{10+\cos x}{11}-\frac{4 x^{2}}{33}+\frac{x^{4}}{220}-\frac{x^{6}}{13860}+\frac{x^{8}}{1995840} .
\end{aligned}
$$

By putting $n=3$ (so $m=5$ ) in (2.2), we get

$$
\begin{aligned}
& \frac{12+\cos x}{13}-\frac{5 x^{2}}{39}+\frac{x^{4}}{195}-\frac{x^{6}}{10920}+\frac{x^{8}}{1179360}-\frac{x^{10}}{259459200}<\frac{\sin x}{x} \\
& <\frac{14+\cos x}{15}-\frac{2 x^{2}}{15}+\frac{x^{4}}{180}-\frac{x^{6}}{9450}+\frac{x^{8}}{907200}-\frac{x^{10}}{149688000} \\
& +\frac{x^{12}}{46702656000} .
\end{aligned}
$$

In the following theorem we prove that the new bounds in Theorem 2.2 are finer than the corresponding bounds in Statement 3.

Theorem 2.3. If $m=2 n-1$ where $n \in \mathbb{N}$ and $x \in(0, \pi)$ then we have

$$
F(x)<\Phi(x) ; \Psi(x)<G(x),
$$

where $F(x), G(x), \Phi(x)$ and $\Psi(x)$ are defined as in Statement 3 and Theorem 2.2.

Proof. Let us prove the first inequality of (2.8). The proof of the second inequality follows a similar line. Let us set

$$
\begin{aligned}
g(x) & =\Phi(x)-F(x) \\
& =\frac{2-2 \cos x}{(2 m+1)(2 m+3)}+\frac{2}{(2 m+1)(2 m+3)} \sum_{k=1}^{m+1} \frac{1}{(2 k) !}(-1)^{k} x^{2 k} .
\end{aligned}
$$

Utilizing (1.6), we get

where

$$
g(x)=\frac{2}{(2 m+1)(2 m+3)} S,
$$

$$
S=\sum_{k=m+2}^{\infty} \frac{(-1)^{k+1}}{(2 k) !} x^{2 k} .
$$

Now $S$ can be viewed as a rest of the alternating series

$$
S^{*}=\sum_{k=1}^{\infty} \frac{(-1)^{k+1}}{(2 k) !} x^{2 k}
$$

which is clearly convergent. Hence $g(x)>0$ which proves the result.

\section{Conclusion}

We established very sharp trigonometric-polynomial bounds of sinc function. New inequalities are refinements of those in (1.2), (1.3) and (1.4). Again by comparing the particular cases of Theorem 2.2 with the corresponding particular cases of Statement 3, it is clear that the polynomial parts in bounds of (2.2) have degrees less than the degrees of corresponding polynomial parts in bounds of (1.4). So the new proposed bounds are not only sharper but also better. These sharp estimates may be used in approximation theory.

Acknowledgments. We thank the two reviewers for their careful reading of the manuscript and their constructive remarks. 


\section{References}

[1] Weisstein E. W.: Sinc Function. In MathWorld-A Wolfram Web Resource, (2019). Retrieved from http://mathworld.wolfram.com/SincFunction.html

[2] Huygens C.: Oeuvres Completes. Société Hollondaise des Sciences, Haga 1888-1940.

[3] Bagul Y. J., Chesneau C: Refined forms of Oppenheim and Cusa-Huygens type inequalities. Preprint hal-01972893v2 (2019). https://hal.archives-ouvertes.fr/hal-01972893/

[4] Malešević B., Lutovac T., Rašajski M., Mortici C.: Extensions of the natural approach to refinements and generalizations of some trigonometric inequalities. Advances in Difference Equations. 2018:90 (2018). https://advancesindifferenceequations.springeropen.com/articles/10.1186/s13662-018-1545-7

[5] Mortici C.: The natural approach of Wilker-Cusa-Huygens Inequalities. Math. Inequal. Appl. 14(3), 535-541 (2011). http:/ /dx.doi.org/10.7153/mia-14-46

[6] Neuman E., Sándor J.: On some inequalities involving trigonometric and hyperbolic functions with emphasis on the Cusa-Huygens, Wilker and Huygens inequalities. Math. Inequal. Appl. 13(4), 715-723 (2010). http:/ /dx.doi.org/10.7153/mia-13-50

[7] Mitrinovic D. S.: Analytic Inequalities. Springer-Verlag, Berlin (1970).

[8] Bhayo B. A., Klén R., Sándor J. New Trigonometric and Hyperbolic Inequalities. Miskolc Mathematical Notes. 18(1), 125-137 (2017). https:/ / doi.org/10.18514/MMN.2017.1560

[9] Sándor J.: Sharp Cusa-Huygens and related inequalities. Notes on Number Theory and Discrete Mathematics. 19(1), 50-54 (2013). http:/ / nntdm.net/volume-19-2013/number-1/50-54/

[10] Bagul Y. J., Chesneau C.: Some new simple inequalities involving exponential, trigonometric and hyperbolic functions. Cubo, A Mathematical Journal. 21(1), 21-35 (2019). http:/ /dx.doi.org/10.4067/S0719-06462019000100021

[11] Malešević B., Makragić M.: A method for proving some inequalities on mixed trigonometric polynomial functions. J. Math. Inequal. 10(3), 849-876 (2016). http:/ / dx.doi.org/10.7153/jmi-10-69

[12] Nenezić M., Zhu L.: Some improvements of Jordan-Steckin and Becker-Stark inequalities. Appl. Anal. Discrete Math. 12(1), 244-256 (2018). https:/ / doi.org/10.2298/AADM1801244N

\section{Affiliations}

RAMKRISHNA M. DHAIGUDE

ADDRESS: Government Vidarbha Institute of Science and Humanities, Department of Mathematics, Amravati (M. S.) - 444604, India.

E-MAIL: rmdhaigude@gmail.com

ORCID ID: 0000-0001-8510-5271

\section{CHRISTOPHE CHESNEAU}

AdDress: University of Caen, LMNO, Normandie, France.

E-MAIL: christophe.chesneau@unicaen.fr

ORCID ID: 0000-0002-1522-9292

YOGESH J. BAGUL

AdDress: K. K. M. College, Manwath(affiliated to SRTMU, Nanded), Department of Mathematics, Dist: Parbhani (M. S.) - 431505, India.

E-MAIL: yjbagul@gmail.com

ORCID ID: 0000-0002-8331-3920 